\title{
ACCELERATION OF THE POLARIZED PROTON BEAM IN THE COOLER SYNCHROTRON COSY
}

\author{
A. Lehrach $^{*}$, U. Bechstedt, J. Dietrich, R. Gebel, K. Henn, R. Maier, D. Prasuhn, A. Schnase, \\ R. Stassen, H. Stockhorst, R. Tölle, Forschungszentrum Jülich, Germany
}

\section{Abstract}

A concept has been developed and realized to accelerate vertically polarized protons in the COoler SYnchrotron COSY at the Forschungszentrum Jülich up to the maximum momentum of $3300 \mathrm{MeV} / \mathrm{c}$ [1]. In addition, different Siberian snake schemes for COSY have been investigated to deliver a longitudinally polarized beam.

\section{INTRODUCTION}

The COoler SYnchrotron and storage ring COSY at the Forschungszentrum Jülich accelerates protons to momenta between $600 \mathrm{MeV} / \mathrm{c}$ and $3300 \mathrm{MeV} / \mathrm{c}$ [2]. At present the beam is used at four internal and three external target places. In addition, a polarized beam can be produced and accelerated at COSY. A colliding beams source, developed by a collaboration of the universities of Bonn, Erlangen, and Cologne is in operation [3]. The polarized $H^{-}$ beam delivered by this source is pre-accelerated in a cyclotron to $295 \mathrm{MeV} / \mathrm{c}$ and injected via stripping injection into the COSY ring. The polarization of the circulating proton beam in COSY is measured continuously during acceleration with the internal EDDA detector [4].

\section{DEPOLARIZING RESONANCES}

For an ideal planar circular accelerator with a vertical guide field the particle spin vector precesses around the vertical axis. Thus the vertical beam polarization is preserved. The number of spin precessions per revolution of the beam in the ring is given by $\nu_{s p}=\gamma G$ [6], where $G=1.7928$ is the proton anomalous magnetic moment and $\gamma$ is the Lorentz factor. During acceleration of a polarized beam, depolarizing resonances are crossed if the precession frequency $\gamma G$ of the spin is equal to the frequency of the encountered spin-perturbing magnetic fields. A strongfocusing synchrotron like COSY has two different types of strong depolarizing resonances, namely imperfection resonances caused by magnetic field errors and misalignments of the magnets and intrinsic resonances excited by horizontal fields due to the vertical focusing.

\section{IMPERFECTION RESONANCES AT COSY}

In the energy range of COSY, five imperfection resonances have to be crossed (Table 1). The resonance strength depends on the vertical closed orbit deviation. A spin flip occurs at all resonances, but the influence of synchrotron

\footnotetext{
*Email: lehrach@bnl.gov, present address: BNL, Upton, NY 11973
}

Table 1: Resonance strength $\epsilon_{r}$ and ratio of preserved polarization $P_{f} / P_{i}$ at imperfection resonances for a typical vertical orbit deviation $y_{c o}^{r m s}$, without considering synchrotron oscillation.

\begin{tabular}{|c||c|c|c|c|c|}
\hline$\gamma G$ & $\begin{array}{c}E_{k i n} \\
(\mathrm{MeV})\end{array}$ & $\begin{array}{c}P \\
(\mathrm{MeV} / \mathrm{c})\end{array}$ & $\begin{array}{c}y_{c o}^{r m s} \\
(\mathrm{~mm})\end{array}$ & $\begin{array}{c}\epsilon_{r} \\
\left(10^{-3}\right)\end{array}$ & $P_{f} / P_{i}$ \\
\hline \hline 2 & 108.4 & 463.8 & 2.3 & 0.95 & -1.00 \\
\hline 3 & 631.8 & 1258.7 & 1.8 & 0.61 & -0.88 \\
\hline 4 & 1155.1 & 1871.2 & 1.6 & 0.96 & -1.00 \\
\hline 5 & 1678.5 & 2442.6 & 1.6 & 0.90 & -1.00 \\
\hline 6 & 2201.8 & 2996.4 & 1.4 & 0.46 & -0.58 \\
\hline
\end{tabular}

oscillation during resonance crossing cannot be neglected. At the first imperfection resonance, the calculated polarization with a typical momentum spread of $\Delta p / p=1 \cdot 10^{-3}$ and a synchrotron tune of $\nu_{s}=1 \cdot 10^{-3}$ is about $P_{f} / P_{i}{ }^{1}$ $=-0.60$. At the other imperfection resonances, the effect is smaller, due to lower momentum spread and synchrotron tune at higher energies. Vertical correction dipoles or a partial snake can be used to preserve polarization at imperfection resonances by exciting adiabatic spin flips. Simulations indicate that an excitation of the vertical orbit by $1 \mathrm{mrad}$ or a rotation angle of the spin in the partial snake of less than $1^{\circ}$ is sufficient to cause a spin flip without depolarization. The vertical correcting dipoles in COSY are designed to increase the vertical orbit deviation by $1 \mathrm{mrad}$ at maximum momentum. The solenoids of the electron cooler system inside COSY are available for use as a partial snake. They are able to rotate the spin around the longitudinal axis by about $8^{\circ}$ at the maximum momentum of COSY.

\section{INTRINSIC RESONANCES AT COSY}

The number of intrinsic resonances depends on the superperiodicity $P$ of the lattice, which is given by the number of identical periods of the accelerator. COSY is a synchrotron with a racetrack design consisting of two $180^{\circ}$ arc sections connected by straight sections. The straight sections can be tuned as telescopes with 1:1 imaging, giving a $2 \pi$ phase advance. Both arcs are composed of three unit cells which are each mirror-symmetrical. A half-cell has a QD-bendQF-bend structure. When the betatron phase advance in the two straight sections of COSY is matched to $2 \pi$, these sections are optically transparent and only the arcs contribute to the strength of intrinsic resonances. One obtains for the

\footnotetext{
${ }^{1}$ Ratio of beam polarization before (i) and after (f) crossing a depolarizing resonance.
} 
resonance condition $\gamma G=k \cdot P \pm\left(\nu_{y}-2\right)$, where $k$ is an integer and $\nu_{y}$ is the vertical betatron tune. The magnetic structure in the arcs allows adjustment of the superperiodicity to $P=2$ or 6 . The corresponding intrinsic resonances in the momentum range of COSY are listed in Table 2.

Table 2: Resonance strengths $\epsilon_{r}$ of intrinsic resonances for a normalized emittance of $1 \pi \mathrm{mm}$ mrad and a vertical working point of 3.61 for different superperiodicities $P$.

\begin{tabular}{|c|c||c|c|c|}
\hline$P$ & $\gamma G$ & $\begin{array}{c}E_{k i n} \\
(\mathrm{MeV})\end{array}$ & $\begin{array}{c}P \\
(\mathrm{MeV} / \mathrm{c})\end{array}$ & $\begin{array}{c}\epsilon_{r} \\
\left(10^{-3}\right)\end{array}$ \\
\hline \hline 2 & $6-\nu_{y}$ & 312.4 & 826.9 & 0.26 \\
\hline 2 & $0+\nu_{y}$ & 950.7 & 1639.3 & 0.21 \\
\hline 2,6 & $8-\nu_{y}$ & 1358.8 & 2096.5 & 1.57 \\
\hline 2 & $2+\nu_{y}$ & 1997.1 & 2781.2 & 0.53 \\
\hline 2 & $10-\nu_{y}$ & 2405.2 & 3208.9 & 0.25 \\
\hline
\end{tabular}

\subsection{Optimizing the optics for polarized beam}

To optimize the optics for a polarized beam, phase advances and betatron amplitudes have been determined along the ring. The measurements were done by exciting continuous betatron oscillations and observing the beam response with a network analyzer between a pair of beam position monitors. With the phase advance of the straight sections matched to $2 \pi$, the superperiodicity of the COSY lattice is determined by the arcs. The superperiodicity equals 6 if all unit cells operate with the same quadrupole settings. In this case only one intrinsic resonance $\gamma G=8-\nu_{y}$ occurs, but the transition crossing takes place at about $1600 \mathrm{MeV} / \mathrm{c}$. To accelerate the beam to maximum momentum, the strength of the horizontally focusing quadrupoles in the inner unit cells is enhanced by about $40 \%$ to shift the transition energy above the maximum momentum. At the same time, the strength of the horizontally focusing quadrupoles in the outer unit cells is decreased by $20 \%$ to keep the betatron tunes constant. The superperiodicity of these beam optics is 2. Consequently, four additional intrinsic resonances are introduced (Table 2 ), which can be suppressed if the harmonics of the corresponding spin-perturbing fields are corrected. Theoretical studies of the COSY lattice revealed the possibility of suppressing the strength of intrinsic resonances using the vertically focusing quadrupoles in the inner unit. This has been confirmed by measurements with a polarized beam [7]. The new method avoids the drawbacks associated with the non-adiabatic nature of tune jumps, which otherwise would be necessary to preserve polarization at all intrinsic resonances at COSY.

\subsection{Successful test of the tune jump system}

A tune jump allows one to preserve polarization at intrinsic resonances by increasing the crossing speed significantly.
This is accomplished by abruptly changing the vertical betatron tune during resonance crossing in the range of microseconds. A magnet system consisting of two pulsed air core quadrupoles was developed. This system was designed to achieve polarization losses of less than $5 \%$ at the strongest intrinsic resonance, and less than $1 \%$ at all other intrinsic resonances in COSY [8]. To meet this goal, a vertical tune jump of 0.06 in $10 \mu \mathrm{s}$ was needed. Fig. 1 shows the polarization of the COSY beam measured during acceleration around the strongest intrinsic resonance $\gamma G=8-\nu_{y}$. This resonance excites a natural spin flip. The polarization loss depends on the vertical emittance of the beam. With a tune jump, the polarization was almost preserved. This tune jump method can be extended to all

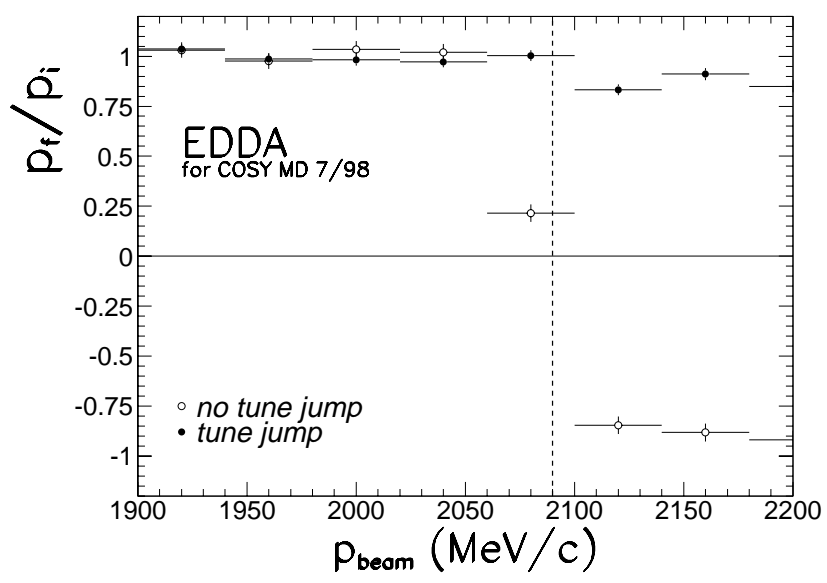

Figure 1: Ratio of preserved beam polarization $P_{f} / P_{i}$ after crossing the strongest intrinsic resonance at $2090 \mathrm{MeV} / \mathrm{c}$ with and without tune jump measured during acceleration with the EDDA detector.

other intrinsic resonances because they are nearly a factor three weaker than the strongest resonance.

\section{ACCELERATION OF THE POLARIZED BEAM}

During the July 1998 running period, the polarized beam was accelerated to $2700 \mathrm{MeV} / \mathrm{c}$. The spin was flipped at the imperfection resonances $\gamma G=2,3,4$ and 5 using correcting dipoles. To avoid polarization losses at the first intrinsic resonance $\left(\gamma G=6-\nu_{y}\right.$ at $\left.827 \mathrm{MeV} / \mathrm{c}\right)$, the acceleration of the beam started with $P=6$ optics. At about $900 \mathrm{MeV} / \mathrm{c}$, the COSY beam optics was then switched to superperiodicity $P=2$ to shift the transition energy. As expected, crossing $\gamma G=0+\nu_{y}$ at $1640 \mathrm{MeV} / \mathrm{c}$ led to polarization losses. After suppressing the strength of intrinsic resonances using the vertically focusing quadrupoles in the inner unit, the ratio of the preserved polarization at the second intrinsic resonance could be significantly increased. At the strongest intrinsic resonance, the polarization could be almost preserved using a tune jump. The measured polarization after this optimization for polarized beam is shown in Fig. 2. In the following running period in December 1998 we were 


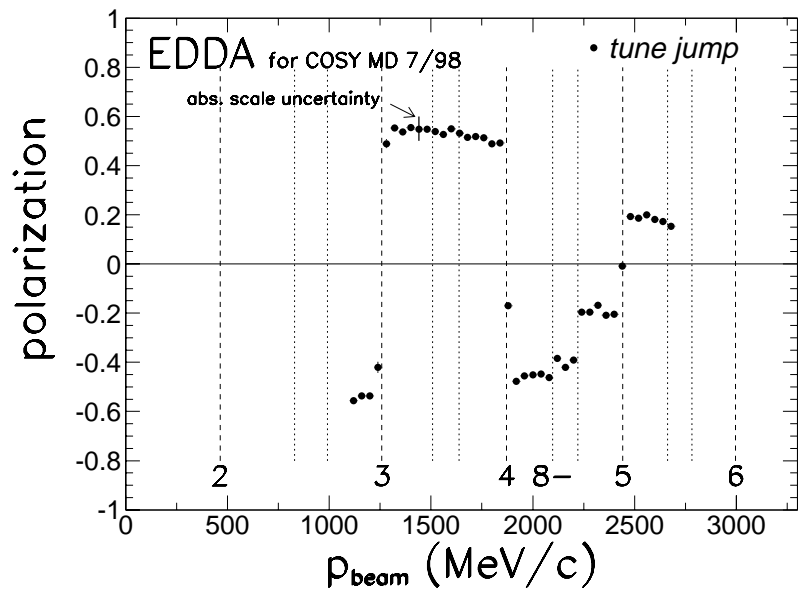

Figure 2: Beam polarization measured during acceleration with the EDDA detector in the momentum range between $1100 \mathrm{MeV} / \mathrm{c}$ and $2700 \mathrm{MeV} / \mathrm{c}$.

able to preserve polarization at the maximum momentum of COSY.

\section{SIBERIAN SNAKE FOR COSY}

Siberian snakes are used to eliminate depolarizing resonances in circular accelerators. The spin is rotated by $180^{\circ}$ in the snake, forcing the spin tune to be a half integer, independent of the beam energy [9]. To conserve the polarization using a Siberian snake with a solenoid field, the direction of the spin vector has to be longitudinal at the symmetry point of the snake, which is point in the accelerator opposite to the position of the snake. This can be achieved by turning on the snake after injection and ramping it until the rotation angle of the spin is $180^{\circ}$. During this process, the spin direction changes from vertical to longitudinal at the symmetry point, and the spin tune changes from $\nu_{s p}=\gamma G$ (without snake) to the nearest half-integer tune ( $180^{\circ}$ snake). To avoid crossing depolarizing resonances, the snake can be turned on at half-integer spin tune. Then the spin tune is equal to a half integer for any snake strength. This condition is satisfied whenever the kinetic energy $E_{k i n}$ is given by $E_{k i n}=370 \mathrm{MeV}+k \cdot 523 \mathrm{MeV}$, where $k$ is an integer. In the energy range of COSY, only a solenoid field is suitable. A solenoid field for $180^{\circ}$ spin rotation rotates the transversal phase space by $32.2^{\circ}$. This can be compensated with two skewed quadrupole doublets. Different snake schemes for COSY have been investigated [1]. One possible magnet arrangement consists of four skewed quadrupoles, with a maximum gradient fields of $34.2 \mathrm{~T} / \mathrm{m}$ and $-32.2 \mathrm{~T} / \mathrm{m}$ rotated by 21.5 and $15.2^{\circ}$ in each doublet, and one solenoid lying in between. The required integral field strength for the solenoid is $12.4 \mathrm{Tm}$ at $3300 \mathrm{MeV} / \mathrm{c}$. Superconducting magnet technology has to be used to achieve an acceptable length of the snake. The total length of such a magnet system is $5.6 \mathrm{~m}$, and it would fit into one of the straight sections of COSY.

\section{CONCLUSION}

Correction dipoles and the solenoids of the electron cooler acting as a partial snake were successfully used to preserve the polarization by exciting adiabatic spin flips. Both methods are available for all five imperfection resonances in the momentum range of COSY. With the standard optics of COSY, five intrinsic resonances are excited. Calculations predict, and measurements confirm, that some of these resonances can be suppressed by changing the optics during acceleration. A tune jump is necessary for the remaining intrinsic resonances. A magnet system consisting of two pulsed air core quadrupoles was developed and successfully used. Polarization measurements during acceleration confirm that the developed concept allows the acceleration of a vertically polarized proton beam up to the maximum momentum of COSY. The installation of a Siberian snake could also provide a longitudinally polarized beam in COSY.

\section{ACKNOWLEDGEMENT}

We are indebted to all members of the COSY team and the collaboration of the polarized source for their support. We are especially grateful to the EDDA collaboration for their sophisticated measurement of the beam polarization during acceleration.

\section{REFERENCES}

[1] A. Lehrach, Erarbeitung und Umsetzung eines Konzepts zur Beschleunigung polarisierter Protonen im Kühlersynchrotron COSY, PhD-thesis Universität Bonn (1997), Bericht des Forschungszentrums Jülich Juel-3501.

[2] R. Maier, Cooler Synchrotron COSY Performance and Perspectives, Nucl. Inst. and Meth. A 390 (1997), 1.

[3] P.D. Eversheim et al., The Polarized Ion-Source for COSY, 12th Int. Symp. on High-Energy Spin Physics, Amsterdam (1996), (published in World Scientific Singapore 1997, 306).

[4] V. Schwarz et al., EDDA As Internal High-Energy Polarimeter, 13th Int. Symp. on High-Energy Spin Physics, Protvino (1998), (submitted to World Scientific Singapore).

[5] A. Lehrach et al., Status of Polarized Beam at COSY, 12th Int. Symp. on High-Energy Spin Physics, Amsterdam (1996), (published in World Scientific Singapore 1997, 416).

[6] V. Bargman, L. Michel, V.L. Telegdi, Phys. Rev. Lett. Vol.2 (1959), 435.

[7] A. Lehrach et al., Suppressing Intrinsic Spin Harmonics at the Cooler Synchrotron COSY, submitted to Nucl. Inst. and Meth. section A (1999).

[8] A. Lehrach et al., Tune jumping system for COSY, IKP Annual Report 1996, Bericht des Forschungszentrums Jülich Juel-3200, 263.

[9] Ya.S. Derbenev and A.M. Kondratenko, Part. Accel. Vol.8 (1978), 115. 